\title{
General Psychiatry Efficacy and safety of repetitive transcranial magnetic stimulation for generalised anxiety disorder: A meta- analysis
}

\author{
Huiru Cui, ${ }^{1}$ Lijuan Jiang, ${ }^{1}$ Yanyan Wei, ${ }^{1}$ Wei Li, ${ }^{1}$ Hui Li, ${ }^{1}$ Junjuan Zhu, ${ }^{1}$ \\ Jiaoyan Pang, ${ }^{2}$ Jijun Wang, ${ }^{1,3,4}$ Chunbo Li ${ }^{1,3,4,5}$
}

To cite: Cui H, Jiang L, Wei Y, et al. Efficacy and safety of repetitive transcranial magnetic stimulation for generalised anxiety disorder: A metaanalysis. General Psychiatry 2019;32:e100051. doi:10.1136/ gpsych-2019-100051

- Additional material is published online only. To view please visit the journal online (http://dx.doi.org/10.1136/ gpsych-2019-100051).

$\mathrm{HC}$ and $\mathrm{LJ}$ contributed equally.

Received 11 January 2019

Revised 31 May 2019

Accepted 29 July 2019

Check for updates

(C) Author(s) (or their employer(s)) 2019. Re-use permitted under CC BY-NC. No commercial re-use. See rights and permissions. Published by BMJ.

For numbered affiliations see end of article.

Correspondence to

Dr Chunbo Li, Shanghai, China; licb@smhc.org.cn

\section{ABSTRACT}

Background Pharmacological and conventional nonpharmacological treatments are only moderately effective in treating generalised anxiety disorder (GAD). Recently, repetitive transcranial magnetic stimulation (rTMS) has attracted interest because of its potential therapeutic value.

Aim To investigate the efficacy and safety of rTMS treatment for GAD.

Methods Literature studies published in English or Chinese were screened in 10 electronic databases up to 5 December 2018. The included studies' bias risk was assessed using Cochrane risk of bias assessment tool. Meta-analysis was performed to compute the standardised mean difference (SMD) and risk ratio (RR) along with its 95\% Cls through using RevMan V.5.3. Heterogeneity was inspected by $\mathrm{I}^{2}$ and the $\chi^{2}$ test. We performed subgroup analysis and meta-regression to investigate heterogeneity. We used funnel plot to assess publication bias. We used the GRADE approach to assess the whole quality of evidence.

Results Twenty-one studies, with a total sample size of 1481, were analysed. The risk of bias in most studies included is moderate, the majority of which are lacking of blinding methods of treatment allocation. The treatment had beneficial effects in the rTMS group compared with the control group in mean anxiety score $(\mathrm{SMD}=-0.68$; $95 \% \mathrm{Cl}-0.89$ to -0.46$)$. None of the 21 studies included here reported severe adverse events. As for dropout rates, there are no statistically significant differences between the two groups ( $\mathrm{RR} 1.14,95 \% \mathrm{Cl} 0.72$ to 1.82) or adverse events (RR $0.95,95 \% \mathrm{Cl} 0.77$ to 1.18 ). No particular influence on the heterogeneity of any variable was observed. The risk of publication bias was low. According to the GRADE approach, the evidence levels of primary outcome (treatment effects) and secondary outcomes (acceptability and safety) were rated as 'medium'.

Conclusion The use of rTMS combined with medication treatment may have a significant positive anti-anxiety effect on patients with GAD. However, we should interpret the results cautiously due to the relatively high heterogeneity of the meta-analysis. Future high-quality clinical trials are needed to confirm our results.

\section{INTRODUCTION}

Generalised anxiety disorder (GAD) has the characteristics of chronic, overwhelming anxiety and worry. ${ }^{1}$ In China, GAD has a lifelong prevalence of approximately $3.2 \% .^{2}$ The anxiety, worry or physical symptoms cause impairment in important functionings such as occupational and social functionings, and cost much medical resource. ${ }^{3}$ Standard first-line treatments for GAD include pharmacotherapy and psychotherapy. ${ }^{4}$ However, treatment effects of the standard therapies are not as good as what we have expected, with approximately $50 \%$ of patients remain having residual symptoms. ${ }^{5}$ Therefore, seeking novel treatment options for GAD is of great importance. ${ }^{6}$

Repetitive transcranial magnetic stimulation (rTMS) neuromodulation has merits because it is non-invasive, well tolerated, safe and so forth. There is a growing number of studies in various mental disorders. ${ }^{7-11}$ During the rTMS treatment, the rTMS coil is placed on the scalp, and at the meantime the coil is electrified so that a magnetic field is produced which could penetrate the skull to induce effects. $^{12}$ The modulation effect of rTMS depends on the frequency, either decreasing or increasing cortical excitability. ${ }^{13}$ The prevailing hypothesis is that the aftereffects of high-frequency (usually $5 \mathrm{~Hz}$ or greater) stimulation are excitatory while those of stimulation with low frequency ( $1 \mathrm{~Hz}$ or less) are inhibitory. ${ }^{14}$ The US Food and Drug Administration has approved the high-frequency rTMS stimulation over the left dorsolateral prefrontal cortex (DLPFC) in treating resistant major depressive disorder (MDD) ${ }^{15}$ The anxiety symptoms have also been improved in patients with MDD following the rTMS, suggesting that rTMS may be a potential treatment for anxiety disorder. ${ }^{16}$ 
Previous rTMS studies have shown the potential beneficial effects of rTMS in patients with GAD. Some studies like Bystritsky et al's open-label study ${ }^{17}$ and Diefenbach $e t$ $a l$ s randomised controlled trial (RCT) ${ }^{18}$ demonstrate fair anxiety reduction using low-frequency rTMS stimulation in GAD, whereas another study also shows a therapeutic effect using high-frequency rTMS. ${ }^{19}$ Although the above studies found positive results of rTMS intervention in patients with GAD, they are varied in rTMS parameters and sample sizes are small. Therefore, it is difficult to get consistent conclusions in view of these studies. Recently, quite a few studies, mostly using low-frequency stimulation parameters, have been reported in China. ${ }^{20-37}$ However, the meta-analysis of efficacy and safety of rTMS for GAD is seldom reported, and there is a need for updates. ${ }^{38}$

\section{METHODS}

Search strategy and methods

We searched for objective studies before 5 December 2018. The search terms were "generalized anxiety disorder", "anxiety", "transcranial magnetic stimulation", "TMS" and "rTMS". The Cochrane Library, PubMed, ISI Web of Knowledge, EMBASE and PsycInfo were retrieved. The Chinese search terms were “重复经颅磁刺激”, “磁刺激”, “ 经颓磁刺激”, ‘跨颖磁刺激”, “广泛性焦虑障碍” and “焦虑 症'. We searched the following Chinese data libraries: Wanfang Data, Chinese National Knowledge Infrastructure, VIP Information, Huayi-Taiwan data and SinoMed.
Studies were included in accordance with PICOS (Participants, Intervention, Comparison, Outcomes and Study design) inclusion criteria: (1) participants: have a diagnosis of GAD according to one of the following diagnostic criteria: the Diagnostic and Statistical Manual of Mental Disorders, fourth edition (DSM-IV) ${ }^{39}$ the 10th revision of the International Classification of Disease (ICD-10) ${ }^{40}$ the third edition of the Chinese Mental Illness Diagnostic Standard (CCMD-3) ${ }^{41}$ or the MINI-international Neuropsychiatric Interview (MINI); ${ }^{42}$ (2) intervention: used rTMS intervention; rTMS could be combined with drug therapy; (3) comparison: the control group used sham rTMS or received no intervention; (4) outcomes: the primary outcome was rTMS efficacy in decreasing the anxiety symptoms of patients with GAD; the secondary outcomes were acceptability and safety of rTMS; (5) study design: RCT. Studies such as case reports, case series, observational studies, meta-analyses and systematic reviews were not included in this study.

\section{Literature search and data extraction}

Two reviewers (HC and $\mathrm{LJ}$ ) independently performed the literature search using the same search strategy. All retrieved literature was managed using the EndNote X7 software. Literature screening and data extraction were performed as follows: (1) duplicates of retrieved studies were excluded. (2) The studies were screened by checking the titles together with their abstracts and studies found to be inappropriate were excluded. (3) As for the remaining

1936 articles published before Dec 5, 2018 were searched (see methods section for search strategy ):

PubMed (211), the Cochrane Library (106), Web of Knowledge (220), EMBASE (446), Psychinfo (301), CNKI (122), Wanfang (183), VIP Information (154) and SinoMed (193)

1542 duplicates excluded

394 articles: 265 published in English, 129 in Chinese

301 articles were excluded after reading the abstracts

93 unduplicated articles: 41 published in English, 52 in Chinese

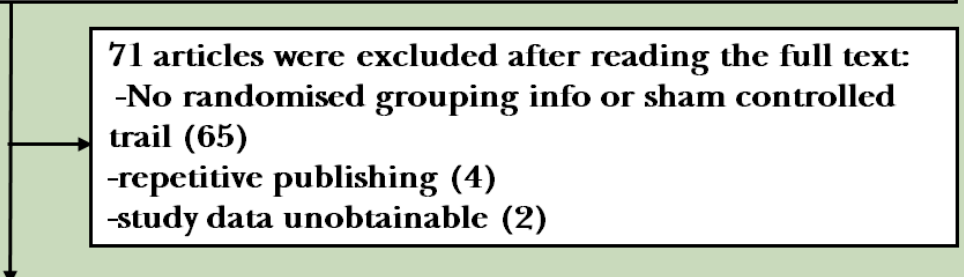

21 unduplicated studies included: 2 published in English, 19 in Chinese

Figure 1 Flowchart of the literature screening. 


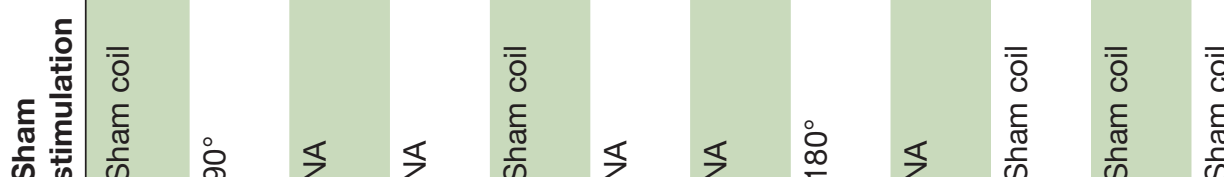




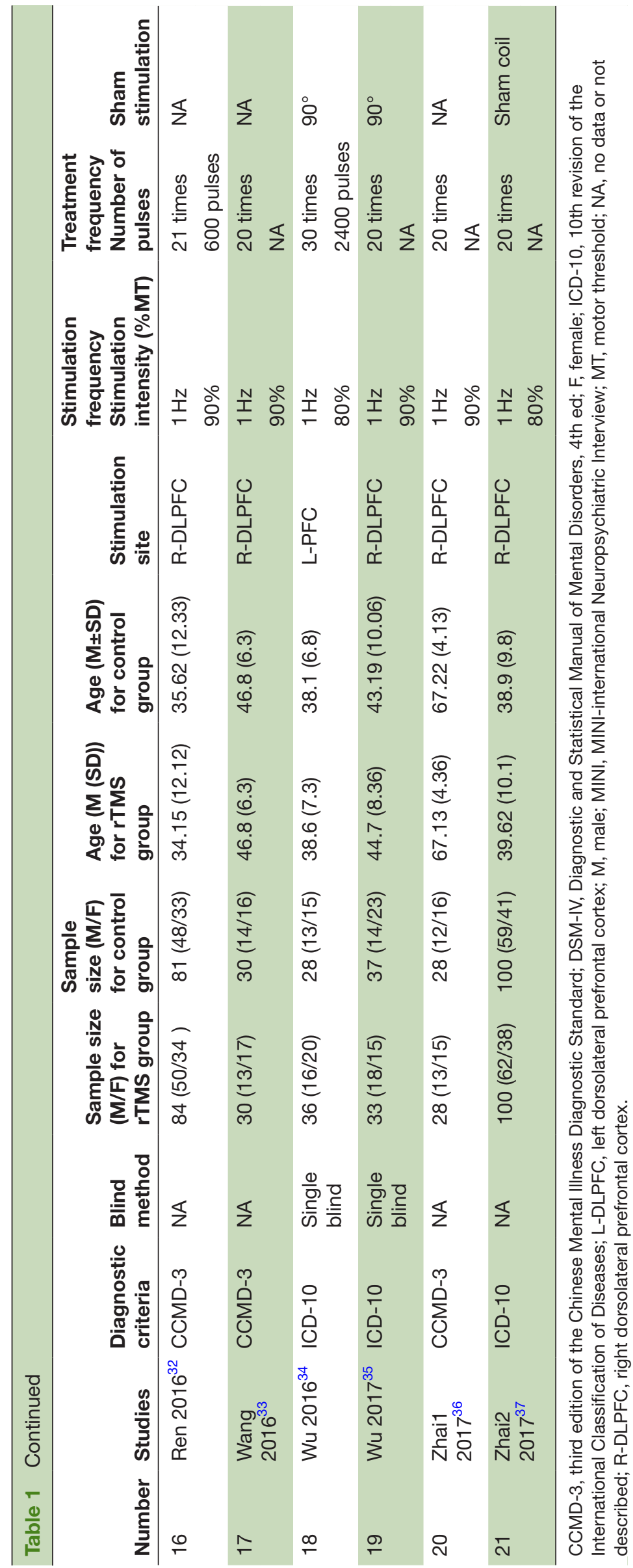

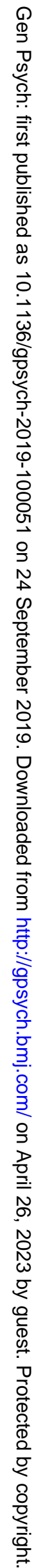




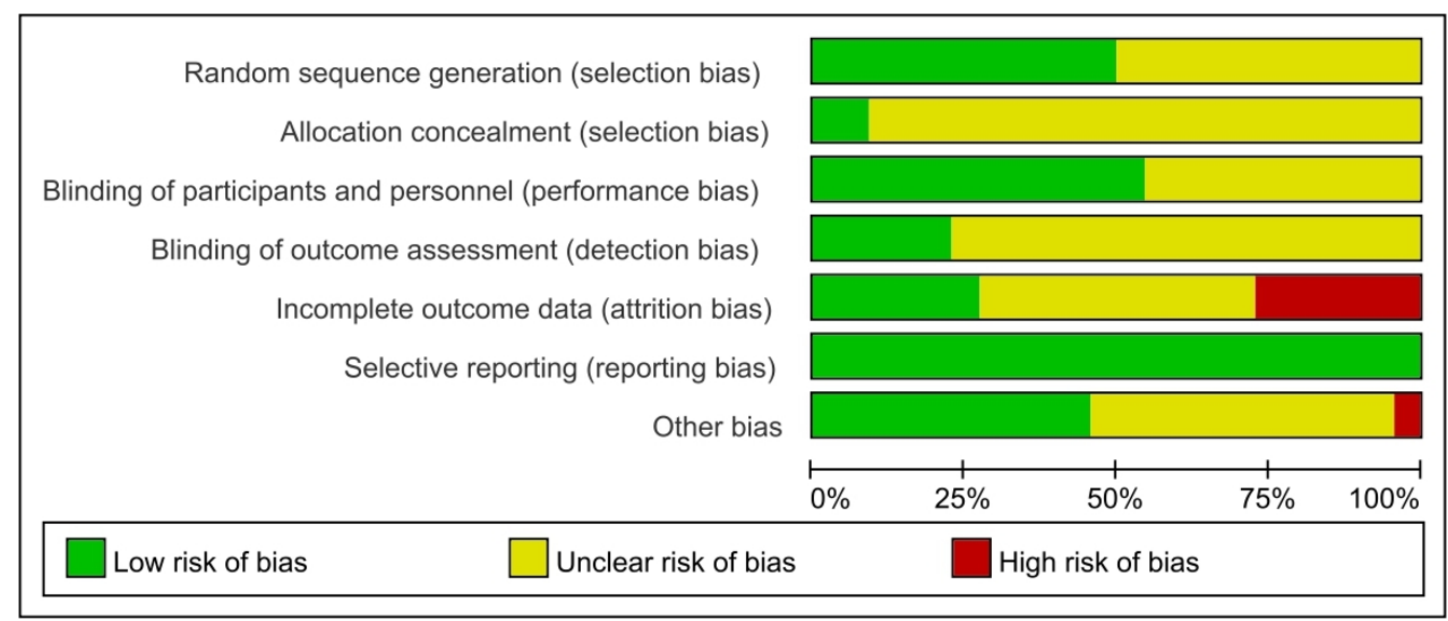

Figure 2 Risk of bias graph: in the form of percentage of each bias risk in all included studies.

literature, full text was checked to confirm their eligibility for inclusion. If the two search results were different, the two researchers reviewed the literature together and analysed the reasons for differences. If the opinions were still inconsistent, a third researcher (HL) would examine the literature and make a final decision. The literature screening process of this study is shown in figure 1 .

The extraction form for included information was developed by HC. Two researchers (LJ and JZ) extracted the relevant data independently. The extracted data included the following items: study author, year of publication, diagnostic criteria, blind method, sample characteristics, stimulation site, stimulation frequency, stimulation intensity based on resting motor threshold, treatment regimen and sham rTMS methods. HRC checked the extraction results.

\section{Quality evaluation of literature}

In accordance with the criteria of bias risk given by the Cochrane handbook, two researchers (HL and WL) independently evaluated the risk of bias. When there was discrepancy between the evaluations of the two researchers, the conclusion would be determined by a third researcher (JP). The specific contents included

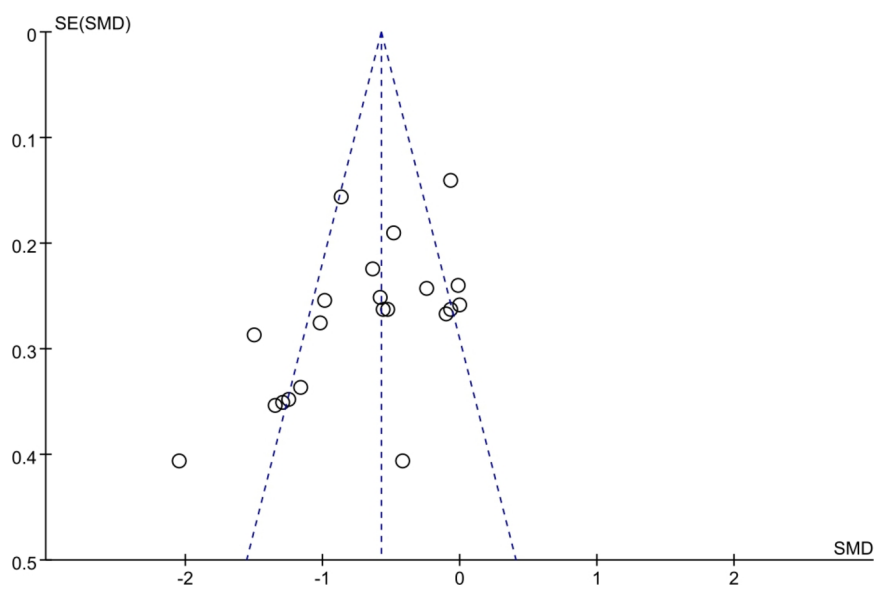

Figure 3 Funnel plot to assess potential publication bias in 21 included studies. SMD, standardised mean difference. the following: (1) random sequence generation (selection bias); (2) allocation concealment (selection bias); (3) blinding of subjects and researchers (performance bias); (4) blinding of outcome data (detection bias); (5) incomplete outcome data (attribution bias); (6) selective reporting (reporting bias); (7) other bias. When the assessment information was lacking, we contacted the corresponding author via email.

\section{Outcome measures}

The primary outcome of this study was rTMS efficacy in decreasing the anxiety symptoms of patients with GAD, which was measured using the reductions of anxiety symptoms assessed by the Hamilton Anxiety Scale (HAMA) ${ }^{43}$

The secondary outcomes: (1) acceptability, which was measured using the dropout rates during the treatment courses. (2) Safety, which was assessed using the number of adverse events like nausea, headache, syncope, insomnia, epilepsy or burned by electrode.

We used Review Manager (RevMan) V.5.3 to compute the combined effects of relative risk (RR), standardised mean deviation (SMD) and CIs. $\mathrm{I}^{2}$ test was used to estimate heterogeneity level $\left(\mathrm{I}^{2}=25 \%\right.$ meant low-level heterogeneity, $50 \%$ medium and $75 \%$ high).$^{44} \mathrm{~A}$ fixed-effects model was used when there was no significant heterogeneity $\left(\mathrm{I}^{2}<50 \%, \mathrm{p} \geq 0.1\right)$, while a random-effects model was selected when heterogeneity was significant $\left(I^{2} \geq 50 \%\right.$, $\mathrm{p}<0.1)$.

\section{Subgroup analysis and meta-regression analysis}

Subgroup analyses were conducted in the left hemisphere versus right hemisphere studies, high frequency $(>1 \mathrm{~Hz})$ versus low frequency $(\leq 1 \mathrm{~Hz})$ studies, and high treatment times ( $>20$ times) versus low treatment times $(\leq 20$ times) studies.

The sources of heterogeneity were explored using meta-regression analysis. Meta-regression analysis was performed using Stata V.15.0 statistical software. ${ }^{45}$ 


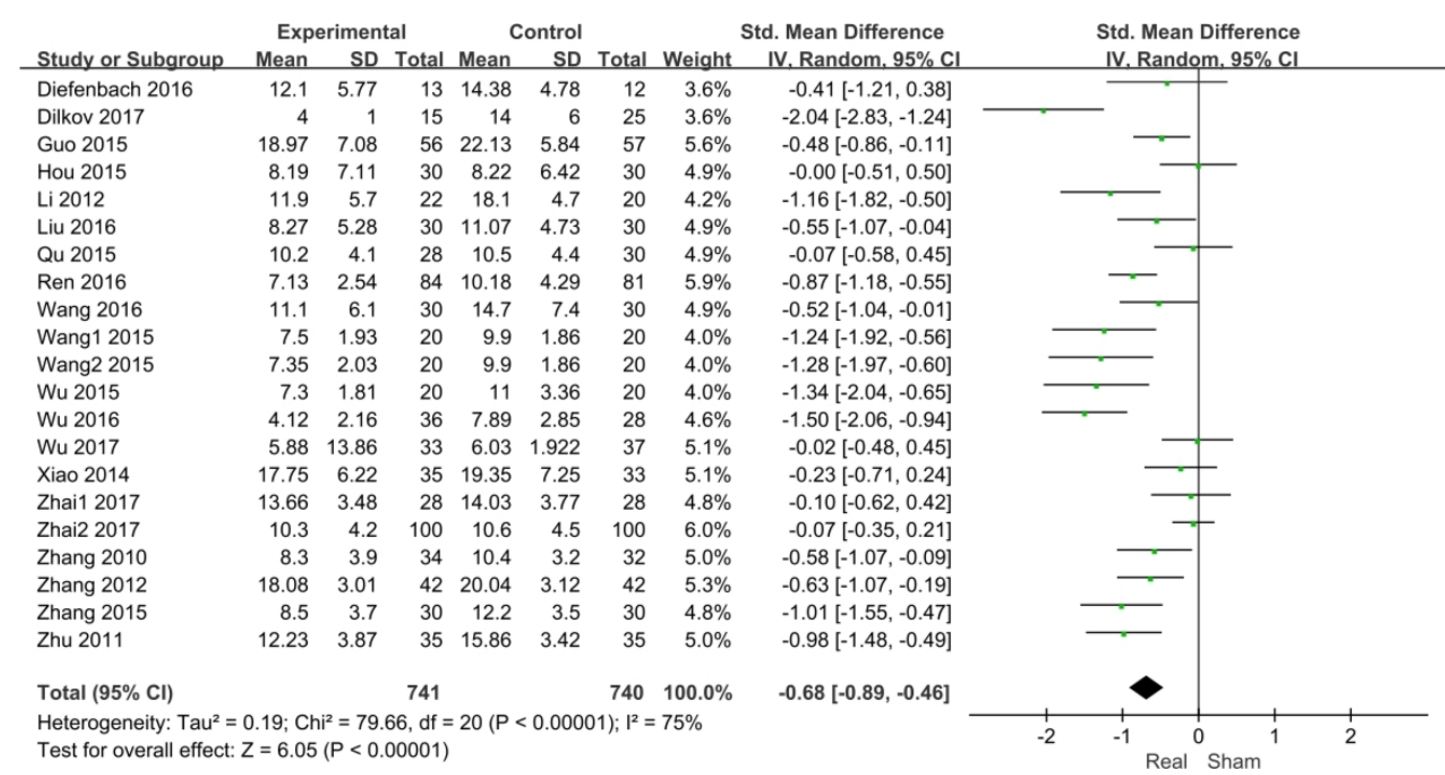

Figure 4 Forest plot illustrating efficacy of repetitive transcranial magnetic stimulation group compared with control group in the treatment of generalised anxiety disorder. Random-effects models were used.

Assessment of reporting biases and sensitivity analysis

The Cochrane funnel plot was used to detect potential publication bias. The planned sensitivity analyses: (1) open-label RCTs will be excluded. (2) The international studies will be excluded.

\section{GRADE assessment}

Quality of evidence was assessed by the Grading of Recommendations Assessment, Development and Evaluation (GRADE) method. ${ }^{46}$ The quality of evidence grades were as follows: (1) high quality, further study was difficult to affect the reliability of the efficacy evaluation results; (2) medium quality, further study was easy to affect the reliability of the efficacy evaluation results and was very likely to change the outcome of the evaluation; (3) low quality, further research was very easy to affect the reliability of the efficacy evaluation results and the evaluation outcome was very likely to change; (4) extremely low quality, the results of any efficacy evaluation were uncertain. Two authors (YW and WL) independently used the GRADE method to rate the overall evidence quality.

\section{RESULTS}

\section{Extracted data of included studies}

In total, 1481 subjects from 21 studies were included in our meta-analysis, which had 732 men and 749 women. One of the 21 studies included only male subjects. ${ }^{47}$ All GAD subjects had to fulfil the diagnostic criteria listed as follows: MINI, ICD-10, CCMD-3 or DSM-IV. In 17 studies, the rTMS treatment was applied to the right hemisphere, including 16 studies where treatment was applied to the right DLPFC and one study to the right parietal lobe. Two studies used high-frequency stimulation, ${ }^{21} 28$ while the other 19 studies used $1 \mathrm{~Hz}$ low-frequency stimulation. The range of stimulation intensity of all included studies was from $80 \%$ to $110 \%$ (of resting motor threshold). As

Table 2 Summary of the quality grade rating of different outcome indicators for the efficacy of rTMS for GAD

\begin{tabular}{|c|c|c|c|c|c|c|c|c|c|}
\hline \multirow[b]{2}{*}{ Outcome indicator } & \multirow{2}{*}{$\begin{array}{l}\text { Number } \\
\text { of } \\
\text { included } \\
\text { cases }\end{array}$} & \multicolumn{2}{|c|}{ Heterogeneity } & \multirow[b]{2}{*}{$\begin{array}{l}\text { Model of } \\
\text { analysis }\end{array}$} & \multicolumn{2}{|c|}{$\begin{array}{l}\text { Group effect } \\
\text { value }\end{array}$} & \multirow[b]{2}{*}{$\begin{array}{l}\text { Esimated } \\
\text { value }\end{array}$} & \multirow[b]{2}{*}{$95 \% \mathrm{Cl}$} & \multirow[b]{2}{*}{ GRADE } \\
\hline & & $\mathrm{I}^{2}$ & $\mathbf{p}$ & & $\mathbf{Z}$ & $\mathbf{p}$ & & & \\
\hline $\begin{array}{l}\text { Treatment effects: } \\
\text { reduction of anxiety } \\
\text { symptoms using HAMA }\end{array}$ & 1481 & $75 \%$ & $<0.001$ & $\begin{array}{l}\text { Random } \\
\text { effect }\end{array}$ & 10.61 & $<0.001$ & -0.68 (SMD) & $\begin{array}{l}-0.89 \text { to }- \\
0.46\end{array}$ & Moderate \\
\hline $\begin{array}{l}\text { Safety of rTMS: adverse } \\
\text { effects, no of adverse } \\
\text { events }\end{array}$ & 1073 & $0 \%$ & 0.49 & Fixed effect & 0.43 & 0.67 & $0.95(\mathrm{RR})$ & 0.77 to 1.18 & Moderate \\
\hline
\end{tabular}

GAD, generalised anxiety disorder; GRADE, Grading of Recommendation Assessment, Development and Evaluation; HAMA, Hamilton Anxiety Scale; RR, relative risk; rTMS, repetitive transcranial magnetic stimulation; SMD, standardised mean difference. 


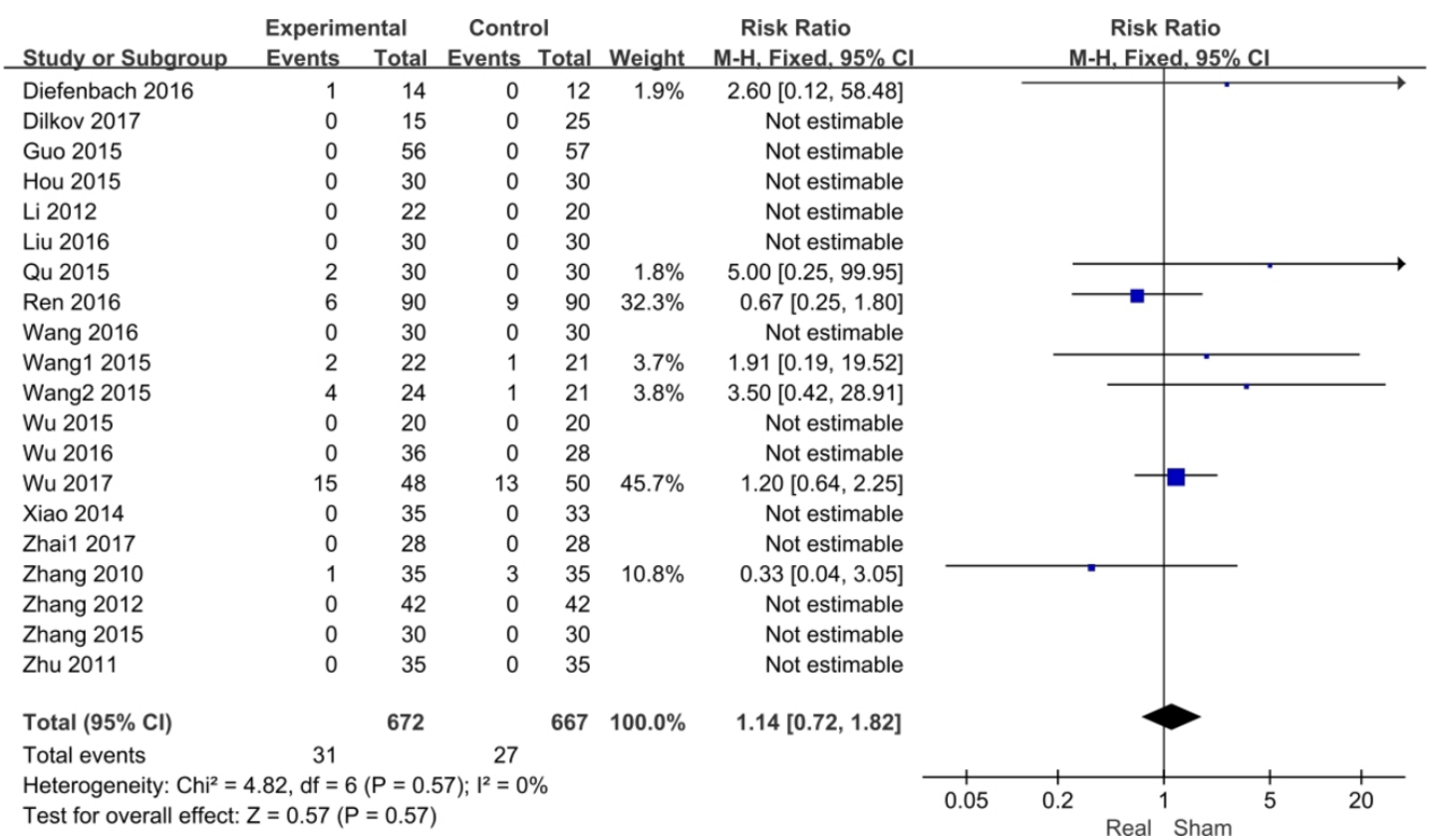

Figure 5 Forest plot illustrating acceptability of repetitive transcranial magnetic stimulation group compared with control group in the treatment of generalised anxiety disorder. Fixed-effects models were used.

for treatment times, the range varied from 10 times to 30 times. As for sham rTMS methods, six studies used sham coils. Four studies rotated the coil by $90^{\circ}$ and one study rotated it by $180^{\circ}$ to achieve the effect of sham therapy. However, 10 studies did not specify how the sham stimulus was provided. Both 'Wang1 2015' and 'Wang2 2015' were from two studies in Wang's master thesis ${ }^{28}$ (table 1 ).

\section{Risk of bias in included studies}

The results of risk of bias are shown in figure 2. As for random sequence generation selection bias, 10 studies were rated as 'low risk'. 18192126282931 35-37 For allocation concealment selection bias, only two studies reported allocated details rating as 'low risk'. ${ }^{18} 19$ For binding of subjects and researchers performance bias, there were six studies reporting binding details and were rated as 'low risk'. ${ }^{18} 19283435$ There were six studies that reported incomplete data due to drop-out and adverse effects, ${ }^{22} 23$ 30 32-34 and they were rated as 'high risk'. For selective reporting bias, all 21 included studies got 'low risk' for none of them reporting results selectively. 'Unclear risk' was given to studies which had unclear information (figure 2).

Furthermore, the funnel plot was quite symmetrical, which suggested the publication bias for included studies was low risk (figure 3).

\section{Effects of interventions}

Efficacy of rTMS treatment

All 21 included studies assessed the efficacy of the rTMS using the HAMA scale (the primary outcome measure).

\begin{tabular}{|c|c|c|c|c|c|c|}
\hline \multirow[b]{2}{*}{ Study or Subgroup } & \multicolumn{2}{|c|}{ Experimental } & \multicolumn{2}{|c|}{ Control } & \multirow[b]{2}{*}{ Weight } & \multirow{2}{*}{$\begin{array}{c}\text { Risk Ratio } \\
\text { M-H. Fixed, } 95 \% \mathrm{Cl}\end{array}$} \\
\hline & Events & Total & Events & Total & & \\
\hline Diefenbach 2016 & 38 & 13 & 24 & 12 & & Not estimable \\
\hline Dilkov 2017 & 4 & 15 & 0 & 25 & $0.3 \%$ & $14.63[0.84,254.00]$ \\
\hline Guo 2015 & 14 & 56 & 20 & 57 & $17.5 \%$ & $0.71[0.40,1.27]$ \\
\hline Hou 2015 & 7 & 30 & 11 & 30 & $9.7 \%$ & $0.64[0.29,1.42]$ \\
\hline Liu 2016 & 14 & 30 & 11 & 30 & $9.7 \%$ & $1.27[0.69,2.33]$ \\
\hline Qu 2015 & 2 & 28 & 4 & 30 & $3.4 \%$ & $0.54[0.11,2.70]$ \\
\hline Wang1 2015 & 4 & 20 & 3 & 20 & $2.6 \%$ & $1.33[0.34,5.21]$ \\
\hline Wang2 2015 & 3 & 20 & 3 & 20 & $2.6 \%$ & $1.00[0.23,4.37]$ \\
\hline Wu 2015 & 4 & 20 & 5 & 20 & $4.4 \%$ & $0.80[0.25,2.55]$ \\
\hline Wu 2016 & 14 & 36 & 12 & 28 & $11.9 \%$ & $0.91[0.50,1.64]$ \\
\hline Wu 2017 & 0 & 33 & 0 & 37 & & Not estimable \\
\hline Xiao 2014 & 12 & 35 & 11 & 33 & $10.0 \%$ & $1.03[0.53,2.00]$ \\
\hline Zhai1 2017 & 6 & 28 & 13 & 28 & $11.5 \%$ & $0.46[0.20,1.04]$ \\
\hline Zhai2 2017 & 4 & 100 & 2 & 100 & $1.8 \%$ & $2.00[0.37,10.67]$ \\
\hline Zhang 2010 & 1 & 34 & 0 & 35 & $0.4 \%$ & $3.09[0.13,73.21]$ \\
\hline Zhu 2011 & 19 & 35 & 16 & 35 & $14.1 \%$ & $1.19[0.74,1.90]$ \\
\hline Total $(95 \% \mathrm{Cl})$ & & 533 & & 540 & $100.0 \%$ & $0.95[0.77,1.18]$ \\
\hline Total events & 146 & & 135 & & & \\
\hline Heterogeneity: $\mathrm{Chi}^{2}=$ & $2.41, \mathrm{df}=$ & $13(P=$ & $0.49) ; 1^{2}=$ & $0 \%$ & & \\
\hline Test for overall effect & $=0.43(P$ & $=0.67)$ & & & & \\
\hline
\end{tabular}

Figure 6 Forest plot illustrating adverse effects of repetitive transcranial magnetic stimulation group compared with control group in treating generalised anxiety disorder. Fixed-effects models were used. 


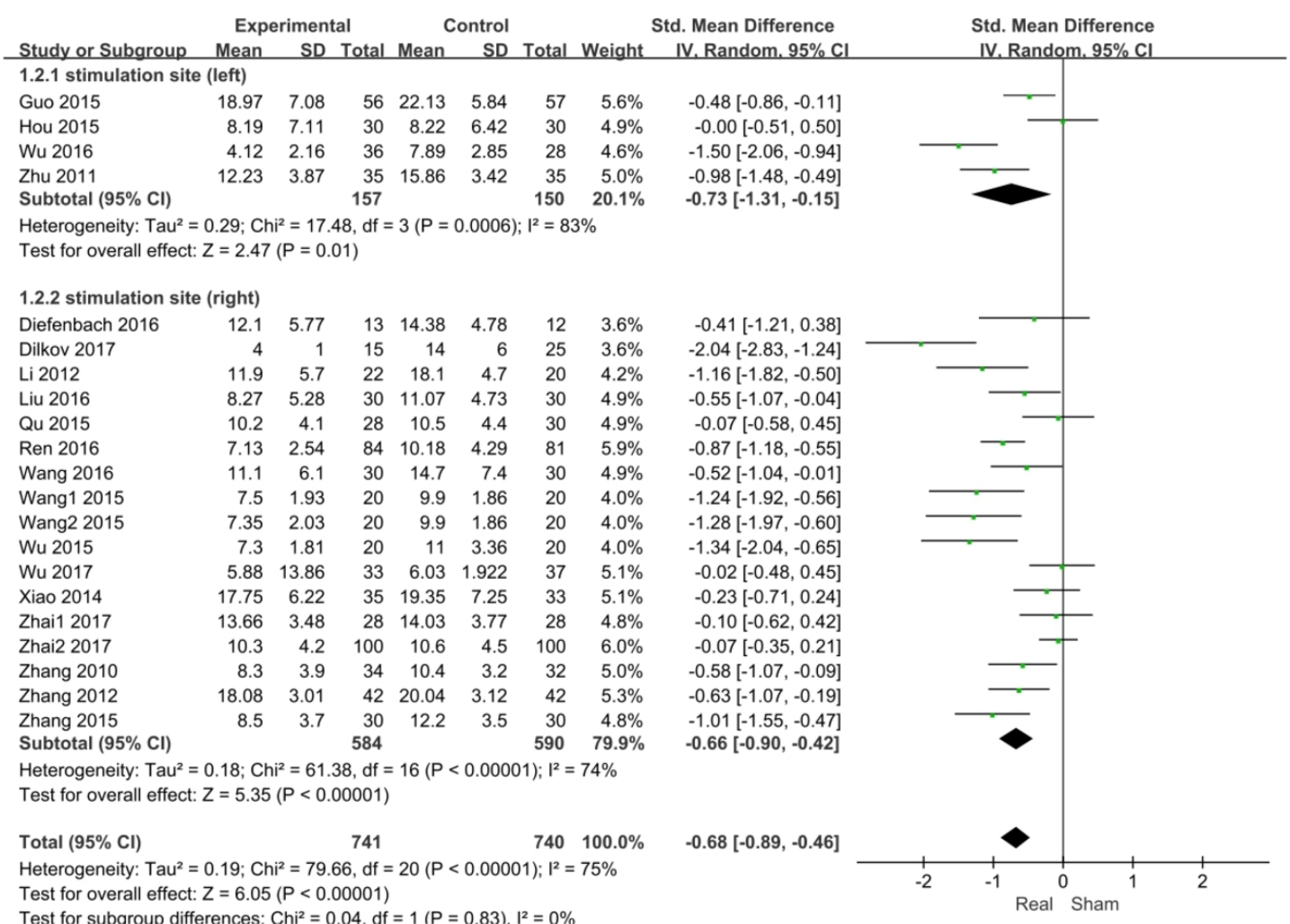

Figure 7 Forest plot of subgroup analysis illustrating efficacy of repetitive transcranial magnetic stimulation group compared with control group in treating generalised anxiety disorder: left hemisphere vs right hemisphere. Random-effects models were used.

The heterogeneity of the included studies was high, that was, $\chi^{2}=79.66, \mathrm{I}^{2}=75 \%$, and random effects model was selected here. Comparisons of the post-treatment HAMA scores indicate that rTMS was an effective treatment in improving anxiety symptoms of GAD ( $\mathrm{SMD}=-0.68$, $95 \% \mathrm{CI}-0.89$ to -0.46$)$, and the difference between treatment group and control group was statistically significant $(\mathrm{Z}=6.05, \mathrm{p}<0.001)$ (figure 4$)$. The overall quality of the evidence of the improvement of anxiety symptoms is 'moderate', according to the GRADE evaluation criteria (table 2).

\section{Acceptability of rTMS treatment}

We analysed the drop-out data from 20 studies which reported drop-outs. The fixed-effects model was selected because of no heterogeneity $\left(\chi^{2}=4.82, \mathrm{I}^{2}=0 \%\right)$. The analysis results found RR was $1.14 \%$ and $95 \% \mathrm{CI}$ was 0.72 to 1.82. There were no significant differences between the rTMS treatment group and control group $(\mathrm{Z}=0.57$, $\mathrm{p}=0.57$ ) (figure 5). The GRADE evidence quality of the outcome was rated as 'moderate' (table 2).

\section{Adverse effects of rTMS treatment}

There were 16 studies that reported adverse effects with a total sample size of 1073 . They reported mild headaches, dizziness, pain in the stimulated area, insomnia and facial spasm. There were no reports of severe adverse events in any included study. The meta-analysis showed that there were no significant differences between the rTMS treatment group and control group
(RR $0.95,95 \%$ CI 0.77 to $1.18, \mathrm{Z}=0.43, \mathrm{p}=0.67$ ). The heterogeneity was quite low; therefore, a fixed-effects model was applied $\left(\chi^{2}=12.41, \mathrm{I}^{2}=0 \%\right.$ ) (figure 6 ). As shown in table 2 , the GRADE evidence quality was rated as 'moderate'.

\section{Subgroup analysis}

The result of the subgroup analysis for rTMS-stimulated sites (left or right hemisphere) was calculated. No significant difference of the effect size of rTMS for GAD was observed betweenthe left hemisphere subgroup $\left(\chi^{2}=17.48, \mathrm{I}^{2}=83 \%, \mathrm{Z}=2.47, \mathrm{p}=0.01\right)$ and right hemisphere subgroup $\left(\chi^{2}=61.38, I^{2}=74 \%, Z=5.35, p<0.001\right)$ (figure 7 ).

Subgroup analysis of stimulated frequency was performed between the subgroup with frequency higher than $1 \mathrm{~Hz}$ (high frequency) and subgroup with frequency lower $\leq 1 \mathrm{~Hz}$ (low frequency). Subgroup analysis showed that no significant difference of the effect size was observed between high frequency subgroup $\left(\chi^{2}=0.36\right.$, $\left.\mathrm{I}^{2}=0 \%, \mathrm{Z}=5.23, \mathrm{p}<0.001\right)$ and low frequency subgroup $\left(\chi^{2}=72.92, \mathrm{I}^{2}=75 \%, \mathrm{Z}=5.42, \mathrm{p}<0.001\right)$ (figure 8$)$.

Subgroup analysis was performed according to the number of treatments, that were the high number of treatments subgroup ( $>20$ times) and low number of treatments subgroup ( $\leq 20$ times). This subgroup analysis revealed a significant difference $(\mathrm{p}=0.03)$ between the high number subgroup $\left(\chi^{2}=29.85, I^{2}=73 \%, Z=5.18\right.$, $\mathrm{p}<0.001)$ and low number subgroup $\left(\chi^{2}=30.87, \mathrm{I}^{2}=64 \%\right.$, $\mathrm{Z}=4.06, \mathrm{p}<0.001$ ) (figure 9). 


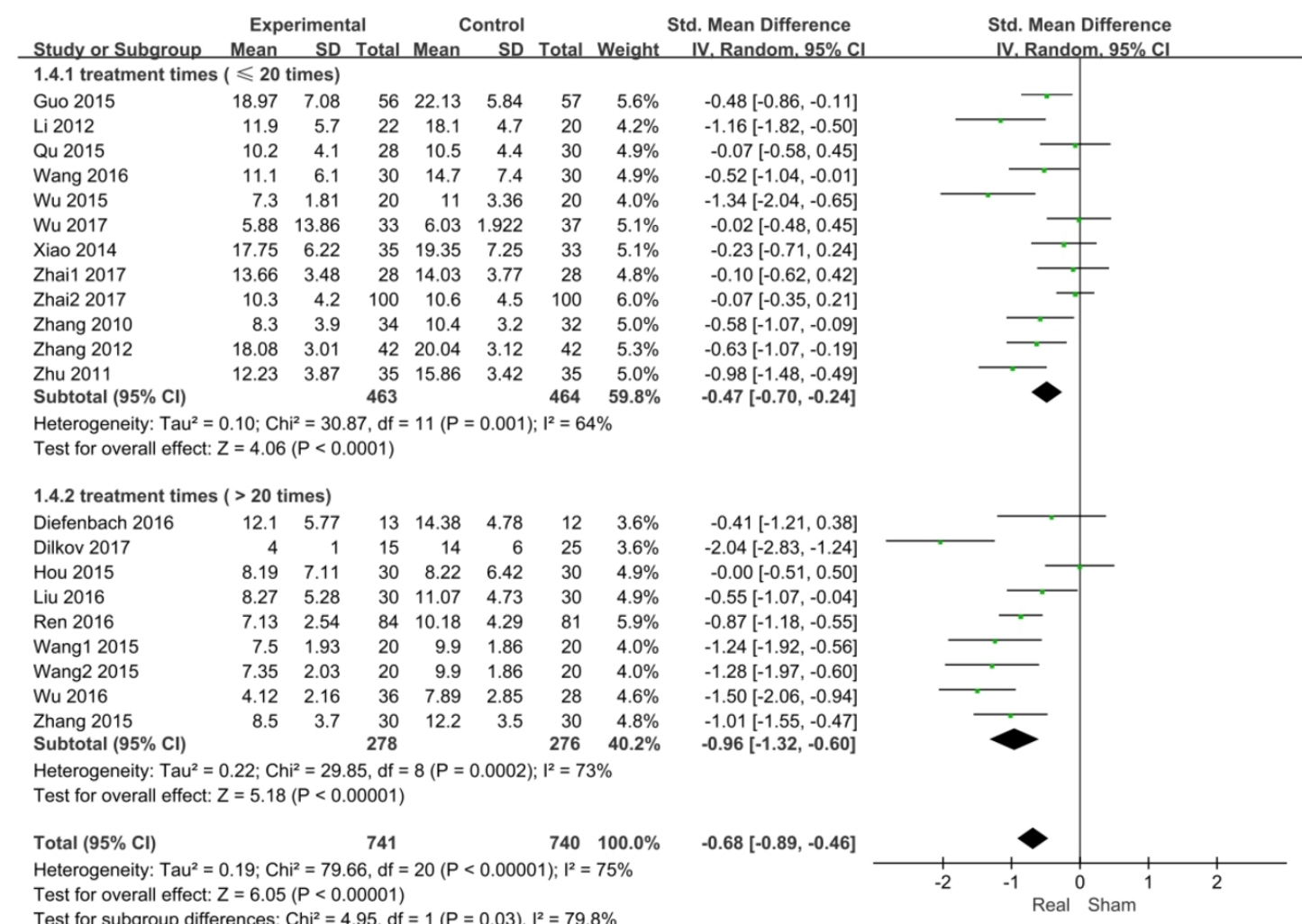

Figure 9 Forest plot of subgroup analysis illustrating efficacy of repetitive transcranial magnetic stimulation group compared with control group in treating generalised anxiety disorder: treatment regimen $\leq 20$ times vs treatment regimen $>20$ times.

Random-effects models were used.

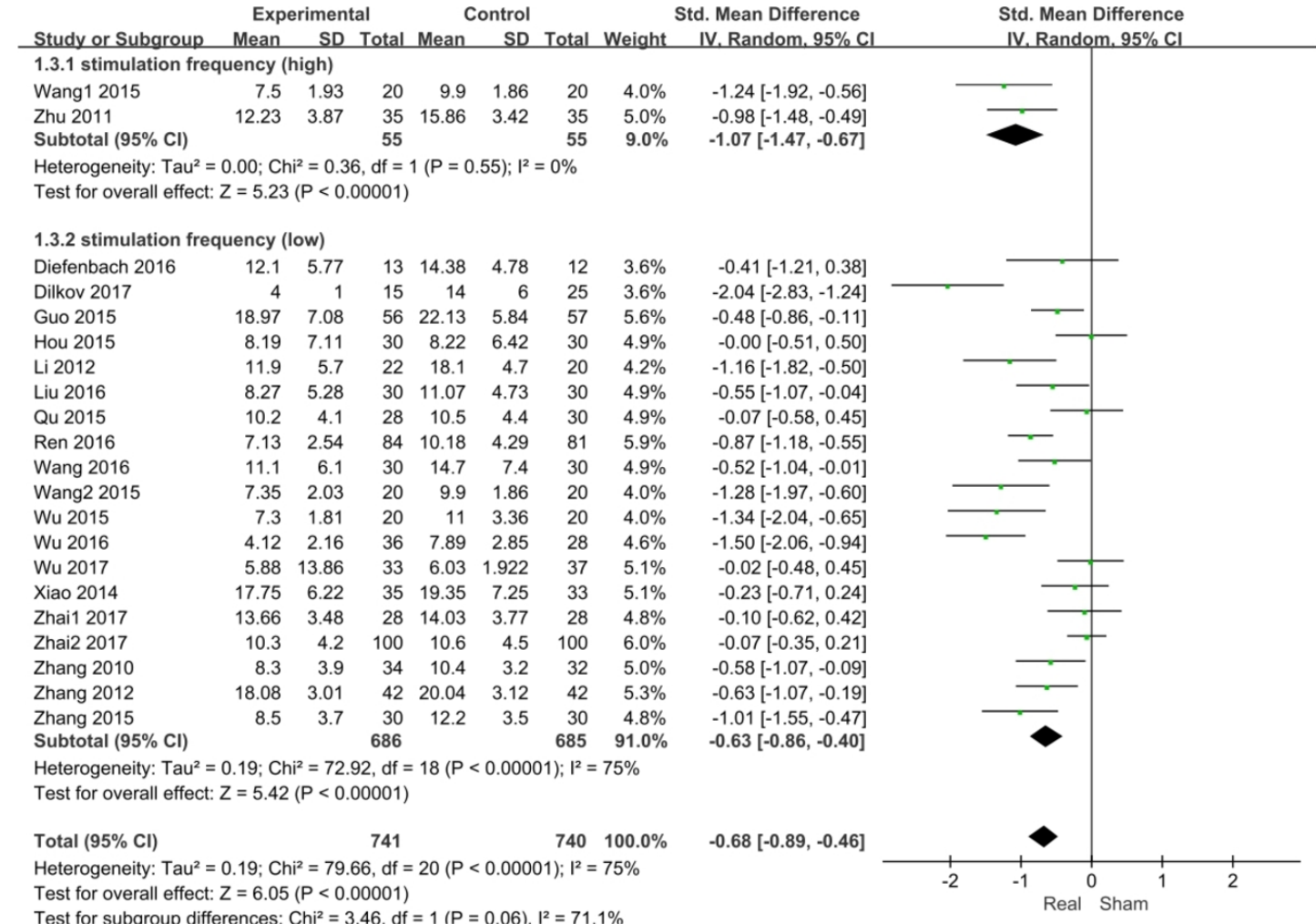

Figure 8 Forest plot of subgroup analysis illustrating efficacy of repetitive transcranial magnetic stimulation group compared with control group in treating generalised anxiety disorder: high frequency stimulation vs low frequency stimulation. Randomeffects models were used. 


\section{Meta-regression analysis}

The heterogeneity of results may be due to differences between studies with respect to patients' baseline symptoms, age and differences in rTMS regimens. However, the meta-regression analysis results indicated that these three variables did not contribute to heterogeneity (table 3).

\section{Sensitivity analysis}

Results of sensitivity analyses demonstrate that the effects and conclusions remained stable when excluding openlabel studies, or international studies, indicating that our results were statistically robust (online supplementary figures S1 and S2).

\section{DISCUSSION}

\section{Main findings}

By integrating Chinese and international research, this meta-analysis has a relatively large sample size of 1481 subjects from 21 studies. Our results suggest that the effect of rTMS plus drug therapy may be better than drug therapy alone in decreasing the anxiety symptoms of GAD.

This meta-analysis found that rTMS might be an effective therapy in decreasing the anxiety symptoms of GAD, which was consistent with other studies. ${ }^{19} 3848$ Moreover, the therapy effects of rTMS still existed in subgroup comparisons in terms of stimulation sites, frequencies and number of treatments with rTMS. In any case, the positive results may be beneficial to improving people's attitudes towards mental illness in the long run. ${ }^{49}$

Furthermore, subgroup analysis by treatment number showed that rTMS had significantly better effects in the high number subgroup than low number subgroup, which suggests the number of rTMS sessions is important to therapeutic effects. However, if the rTMS dosing is too intensive, some patients would refuse and leave. Therefore, developing the optimal, acceptable and feasible dosing treatment of rTMS is of great importance. ${ }^{18}$

In terms of acceptability and adverse effects, rTMS had good acceptability, and no serious adverse effects were found. Moreover, rTMS could be effective for at least two ethnicities since included subjects in our study were

\begin{tabular}{|c|c|c|c|c|c|}
\hline Factor & Coefficient & SE & $\mathbf{t}$ & $P>|t|$ & $95 \% \mathrm{Cl}$ \\
\hline $\begin{array}{l}\text { Baseline } \\
\text { HAMA } \\
\text { score }\end{array}$ & -0.15 & 0.48 & -0.31 & 0.76 & $\begin{array}{l}-1.12 \text { to } \\
0.86\end{array}$ \\
\hline Age & -0.08 & 0.12 & -0.71 & 0.49 & $\begin{array}{l}-0.33 \text { to } \\
0.16\end{array}$ \\
\hline $\begin{array}{l}\text { Treatment } \\
\text { times of } \\
\text { rTMS }\end{array}$ & -0.19 & 0.15 & -1.21 & 0.24 & $\begin{array}{l}-0.51 \text { to } \\
0.14\end{array}$ \\
\hline
\end{tabular}

HAMA, Hamilton Anxiety Scale; rTMS, repetitive transcranial magnetic stimulation. from both Western and Asian countries. Therefore, rTMS appears to be an effective, safe intervention in treating patients with GAD. However, as is well illustrated by this meta-analysis, we need more high-quality studies to contribute to the optimal parameter settings in the future. Note that the overuse of rTMS should be avoided due to the uncertainties about its exact neural mechanism..$^{50-52}$

All studies claimed that they used randomised methods in their studies; however, only two papers reported detailed allocation concealment. As a result, the risk of selection bias is quite high here (figure 2). On the basis of the risk of bias assessment of every study, the evidence quality GRADE rating of the primary outcome indicator (treatment effects) was 'moderate', meaning that the outcome indicator result was a medium recommendation for supporting the use of rTMS intervention. Highquality studies are needed to substantiate the findings in this study.

\section{Limitations}

We should pay attention to some limitations of this meta-analysis. One limitation is the small sample size existing in some of the included studies, which may lead to statistical bias. Another limitation is that our included studies recruited different subjects and adopted different treatment parameters. The differences were reflected in the age and baseline anxiety levels of patients with GAD, and in the rTMS parameters (eg, stimulation site, frequency and regimen). Although all these differences are not sources of heterogeneity based on the subgroup analysis and meta-regression, the existance of robust heterogeneity suggests that we must be cautious about the current conclusion. Moreover, the latest ideas suggest we could use connec${\text { tivity-based } \text { targeting }^{53} \text { or neuronavigation }}^{54}$ to help optimise rTMS' effects. However, none of the Chinese studies included in this meta-analysis use the aforementioned methods to promote the effects of rTMS.

\section{Implications}

This meta-analysis evaluated the effect of rTMS intervention plus drug therapy in treating GAD and found that the use of rTMS had a relative effect on the improvement of anxiety symptoms. Although it had good acceptability and safety, the treatment might induce adverse effects such as dizziness and headache, among others. For patients with GAD who were resistant to traditional treatments, the use of rTMS might be considered clinically. However, we should interpret the results cautiously due to the high heterogeneity of this study.

\footnotetext{
Author affiliations

${ }^{1}$ Shanghai Key Laboratory of Psychotic Disorders, Shanghai Mental Health Center, Shanghai Jiao Tong University School of Medicine, Shanghai, China

${ }^{2}$ School of government, Shanghai University of Political Science and Law, Shanghai, China

${ }^{3}$ Institute of Psychology and Behavioral Science, Shanghai Jiao Tong University, Shanghai, China
} 
${ }^{4}$ Center for Excellence in Brain Science and Intelligence Technology (CEBSIT), Chinese Academy of Science, Shanghai, China

${ }^{5}$ Brain Science and Technology Research Center, Shanghai Jiao Tong University, Shanghai, China

Contributors $\mathrm{HC}$ and LJ were responsible for the literature screening. LJ and JZ were responsible for data extraction. HL, YW, JP and WL were responsible for risk of bias assessment. $\mathrm{HC}$ and $\mathrm{LJ}$ were responsible for statistical analysis and writing up the article. JW and CL were responsible for planning and guidance on this paper.

Funding Shanghai Science and Technology Committee (18411952400, $19411968600,16411965000)$, Shanghai Municipal Natural Science Foundation (18ZR1432600), Shanghai Municipal Commission of Health and Family Planning Foundation (20164Y0215, 20174Y0021),and SHSMU-ION Research Centre for Brain Disorders (2015NKX004).

Competing interests None declared.

Patient consent for publication Not required

Provenance and peer review Not commissioned; externally peer reviewed.

Data availability statement № additional data are available.

Open access This is an open access article distributed in accordance with the Creative Commons Attribution Non Commercial (CC BY-NC 4.0) license, which permits others to distribute, remix, adapt, build upon this work non-commercially, and license their derivative works on different terms, provided the original work is properly cited, appropriate credit is given, any changes made indicated, and the use is non-commercial. See: http://creativecommons.org/licenses/by-nc/4.0/.

\section{REFERENCES}

1 Cui H, Zhang J, Liu Y, et al. Differential alterations of resting-state functional connectivity in generalized anxiety disorder and panic disorder. Hum Brain Mapp 2016;37:1459-73.

$2 \mathrm{Hu}$ Q, Wan Y, Su L, et al. Prevalence of anxiety disorder among mainland residents in China: a meta-analysis. Chin J Psychiatry 2013:46:204-11.

3 Bandelow B, Michaelis S, Wedekind D. Treatment of anxiety disorders. Dialogues Clin Neurosci 2017;19:93-107.

4 Ströhle A, Gensichen J, Domschke K. The diagnosis and treatment of anxiety disorders. Dtsch Arztebl Int 2018;155:611-20.

5 Patterson B, Van Ameringen M. Augmentation strategies for treatment-resistant anxiety disorders: a systematic review and metaanalysis. Depress Anxiety 2016;33:728-36.

6 Kozel FA. Clinical repetitive transcranial magnetic stimulation for posttraumatic stress disorder, generalized anxiety disorder, and bipolar disorder. Psychiatr Clin North Am 2018;41:433-46.

7 Cui H, Li W, Wang J, et al. Repetitive transcranial magnetic stimulation and transcranial direct current stimulation in the treatment of anxiety disorders: a review. J Int Psychiatry 2018;45:983-5.

8 Stip E, Blain-Juste M-E, Farmer O, et al. Catatonia with schizophrenia: from ECT to rTMS. L'Encéphale 2018;44:183-7.

9 Zhang T, Zhu J, Xu L, et al. Add-on rTMS for the acute treatment of depressive symptoms is probably more effective in adolescents than in adults: evidence from real-world clinical practice. Brain Stimul 2019;12:103-9.

10 Zhang T, Sun W, Zhu J, et al. Effect of adjunct repetitive transcranial magnetic stimulation in elderly patients with acute depressive episode: supporting evidence from a real-world observation. Am J Geriat Psych 2019;27:91-2.

$11 \mathrm{Li} \mathrm{H}$, Wang J, Li C, et al. Repetitive transcranial magnetic stimulation (rTMS) for panic disorder in adults. Cochrane Database Syst Rev 2014;144.

12 Terranova C, Rizzo V, Cacciola A, et al. Is there a future for noninvasive brain stimulation as a therapeutic tool? Front Neurol 2018;9:1146.

13 Guo Q, Li C, Wang J. Updated review on the clinical use of repetitive transcranial magnetic stimulation in psychiatric disorders. Neurosci Bull 2017;33:747-56.

14 McClintock SM, Reti IM, Carpenter LL, et al. Consensus recommendations for the clinical application of repetitive transcranial magnetic stimulation (rTMS) in the treatment of depression. J Clin Psychiatry 2018;79:35-48.

15 Connolly KR, Helmer A, Cristancho MA, et al. Effectiveness of transcranial magnetic stimulation in clinical practice post-FDA approval in the United States: results observed with the first 100 consecutive cases of depression at an academic medical center. $J$ Clin Psychiatry 2012;73:e567-73.

16 Diefenbach GJ, Bragdon L, Goethe JW. Treating anxious depression using repetitive transcranial magnetic stimulation. J Affect Disord 2013;151:365-8.

17 Bystritsky A, Kaplan JT, Feusner JD, et al. A preliminary study of fMRI-guided rTMS in the treatment of generalized anxiety disorder. $J$ Clin Psychiatry 2008;69:1092-8.

18 Diefenbach GJ, Bragdon LB, Zertuche L, et al. Repetitive transcrania magnetic stimulation for generalised anxiety disorder: a pilot randomised, double-blind, sham-controlled trial. $\mathrm{Br} J$ Psychiatry 2016;209:222-8.

19 Dilkov D, Hawken ER, Kaludiev E, et al. Repetitive transcranial magnetic stimulation of the right dorsal lateral prefrontal cortex in the treatment of generalized anxiety disorder: a randomized, doubleblind sham controlled clinical trial. Prog Neuropsychopharmacol Biol Psychiatry 2017;78:61-5.

20 Zhang X, Han P, Cheng H, et al. Preliminary study on the early-stage efficacy of repetitive transcranial magnetic stimulation (rTMS) in combination with medication in the treatment of generalized anxiety disorder. J Neurosci Mental Health 2010;10:569-71.

21 Zhu Y, Cai M, Lin M, et al. The efficacy of repetitive transcranial magnetic stimulation combined with paroxetine in the treatment of generalized anxiety disorder. Chin J Phy Med Rehabil 2011;33:125-7.

22 Li Y, Wang Y, Zhan S, et al. A sham-control study of repetitive transcranial magnetic stimulation (rTMS) in the treatment of generalized anxiety disorder (GAD). J Brain Nerv Dis 2012;20:84-8.

23 Zhang D, Zhang D, Shi F. Curative effect of transcranial magnetic stimulation and relaxation on generalized anxiety disorder in children. J Appl Clin Pediatr 2012;15:1191-2.

24 Xiao Z, Gan X, Liu Q, et al. Clinical observation on JiuWer Zhenxin particles combined with transcranial magnetic stimulation in the treatment of generalized anxiety disorder. Chin J Integr Med Cardio Cerebrovasc Dis 2014;5:533-5.

25 Guo L, Cai M, Liu G, et al. Clinical controlled study of repetitive transcranial magnetic stimulation combined with duloxetine versus duloxetine in the treatment of generalized anxiety disorder. J Clin Psych 2015;3:176-8.

$26 \mathrm{Hou} Z$. The effect of duloxetine alone comparison with the united repetitive transcranial magnetic stimulation in the treatment of generalized anxiety disorder. Chin J Prim Med Pharm 2015;21:3308-10.

$27 \mathrm{Qu}$ J, Yuan F. A control study of repetitive transcranial magnetic stimulation in the treatment of generalized anxiety disorder. J Clin Psychosom Dis 2015;2:23-4.

28 Wang X. The effect, safety and cognitive function of different frequency rTMS combined with paroxetine in the treatment of generalized anxiety disorder [硕士]. Xinxiang Medical University, 2015.

29 Wu D, Li Y, He S, et al. Early effect of low frequency repetitive transcranial magnetic stimulation combined with venlafaxine in treatment of patients with generalized anxiety disorder. J Psych 2015;5:351-3.

30 Zhang $\mathrm{C}$, Zhang Y. A control study of paroxetine plus rTMS in generalized anxiety disorders. J Clin Psychosom Dis 2015;1:24-6.

31 Liu X, Ma Y, Li J, et al. Clinical control study of low frequency repetitive transcranial magnetic stimulation combined with escitalopram oxalate in the treatment of generalized anxiety disorder Sichuan Mental Health 2016;2:146-9.

32 Ren J, Ruan H, Hu Y. Clinical effects of repetitive transcranial magnetic stimulation combined with dutoxetine on patients with generalized anxiety disorder. J Hainan Med Univ 2016:22:477-80.

33 Wang K. The clinical research of repetitive transcranial magnetic stimulation combined with JiuWer Zhenxin particles in the treatment of generalized anxiety disorder. Med Inform 2016;21:191-2.

34 Wu X, Liu L, Liang X. Clinical comparative study of generalized anxiety disorder treated with low-frequency repetitive transcrania magnetic stimulation combined with mirtazapine. Prac J Med Pharm 2016:12:1064-6.

$35 \mathrm{Wu} \mathrm{H}$. The clinical research of repetitive transcranial magnetic stimulation combined with venlafaxine and lorazepam in the treatment of generalized anxiety disorder [硕士]. Nanchang University, 2017.

36 Zhai S, Yi G, Liao B, et al. The clinical research of repetitive transcranial magnetic stimulation combined with escitalopram in the treatment of generalized anxiety disorder. Contemp Med 2017:19:119-20.

37 Zhai X, Liu J, Ni A. The efficacy of repetitive transcranial magnetic stimulation in the treatment of generalized anxiety disorder. J Clin Med 2017:91:17907-8. 
38 Li L, Hu W, Gao Y, et al. Efficacy and adverse reactions of repetitive transcranial magnetic stimulation on general anxiety disorder: a meta-analysis. Chin J Behav Med Brain Sci 2016;25.

39 Association AP. Diagnostic and statistical manual of mental disorder. 4th ed. Washington DC: APA, 1994.

40 Dilling H, Dittmann V. [Psychiatric diagnosis following the 10th revision of the International Classification of Diseases (ICD-10)] Nervenarzt 1990;61:259-70.

41 Chinese Medical Association Psychiatry Branch. China classification and diagnostic criteria of mental disorders. Third edition (Mental Disorders), 2001.

42 Pettersson A, Modin S, Wahlström R, et al. The Mini-International neuropsychiatric interview is useful and well accepted as part of the clinical assessment for depression and anxiety in primary care: a mixed-methods study. BMC Fam Pract 2018;19:19.

43 Hamilton M. The assessment of anxiety states by rating. $\mathrm{Br} J$ Med Psychol 1959;32:50-5.

44 Augusteijn HEM, van Aert RCM, van Assen MALM. The effect of publication bias on the $Q$ test and assessment of heterogeneity. Psychol Methods 2019;24:116-134.

45 Stata statistical software: release 15 [computer program]. College Station: StataCorp LLC. 2017.

46 Mendoza C, Kraemer P, Herrera $P$, et al. [Clinical guidelines using the GRADE system (Grading of Recommendations Assessment, Development and Evaluation)]. Rev Med Chil 2017;145:1463-70.

$47 \mathrm{Wu} \mathrm{H}, \mathrm{Hu} \mathrm{M}, \mathrm{Yu} \mathrm{B}$, et al. Repetitive transcranial magnetic stimulation combined with venlafaxine and lorazepam for treatment of generalized anxiety. Shanghai Arch Psych 2016;4:212-7.
48 Assaf M, Rabany L, Zertuche L, et al. Neural functional architecture and modulation during decision making under uncertainty in individuals with generalized anxiety disorder. Brain Behav 2018;8:e01015.

49 Desai R, Panchal B, Vala A, et al. Impact of clinical posting in psychiatry on the attitudes towards psychiatry and mental illness in undergraduate medical students. Gen Psychiatr 2019;32:e100072-111.

50 lannone A, Cruz APdeM, Brasil-Neto JP, et al. Transcranial magnetic stimulation and transcranial direct current stimulation appear to be safe neuromodulatory techniques useful in the treatment of anxiety disorders and other neuropsychiatric disorders. Arq Neuropsiquiatr 2016;74:829-35.

51 Taïb S, Arbus C, Sauvaget A, et al. How does repetitive transcranial magnetic stimulation influence the brain in depressive disorders?: a review of neuroimaging magnetic resonance imaging studies. J Ect 2018;34:79-86.

52 Peng Z, Zhou C, Xue S, et al. Mechanism of repetitive transcranial magnetic stimulation for depression. Shanghai Arch Psychiatry 2018;30:84-92.

53 Ning L, Makris N, Camprodon JA, et al. Limits and reproducibility of resting-state functional MRI definition of DLPFC targets for neuromodulation. Brain Stimul 2019;12:129-38.

54 Wang $\mathrm{H}$, Jin J, Wang $\mathrm{X}$, et al. Non-orthogonal one-step calibration method for robotized transcranial magnetic stimulation. Biomed Eng Online 2018;17:137.

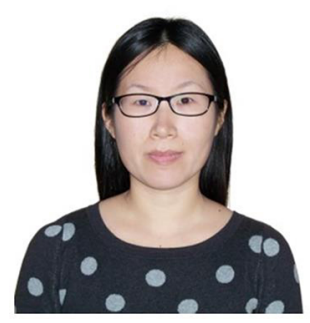

Huiru Cui obtained her Master's degree from Fudan University School of Medicine in 2006. She is currently working as an assistant researcher of the Department of Neuroimaging at Shanghai Mental Health Center. Her main research interests are clinical characteristics and the pathogenesis of anxiety disorders, schizophrenia and other common mental disorders. 